\title{
Putting calories on the menu in Ireland: evaluation of an online calorie calculator for food businesses
}

\author{
S. M. Kelly ${ }^{1}$, M. A. Kerr ${ }^{1}$, T. A. McCaffrey ${ }^{1,2}$, M. B. E. Livingstone ${ }^{1}$, H. McCarthy ${ }^{1}$, C. Groves ${ }^{1}$, \\ N. Browne ${ }^{1}$, F. E. Douglas ${ }^{3}$, S. J. Williams ${ }^{3}$, D. M. O'Connor ${ }^{3}$ and M. A. T. Flynn \\ ${ }^{1}$ Northern Ireland Centre for Food and Health, University of Ulster, Coleraine BT52 1SA, UK, ${ }^{2}$ Department of \\ Nutrition and Dietetics, Monash University, VIC 3168, Australia and ${ }^{3}$ Public Health Nutrition, Food Safety Authority \\ of Ireland, Dublin 1, Republic of Ireland
}

A key challenge to curbing excessive energy intake is the public's significant underestimation of the calorie content of food ${ }^{(1)}$. Calorie labelling on menus (CLM) has been proposed to correct misperceptions and to assist consumers with making more informed choices at point-of-purchase. Recently, Irish consumers and food businesses (FBs) demonstrated support for CLM ${ }^{(2)}$. However, FBs expressed concerns around their lack of expertise in calculating the calorie content of foods sold and the potential costs involved in hiring experts every time menus change. Subsequently an online calorie calculator (MenuCal) was developed by the Food Safety Authority of Ireland (FSAI) to be used by FBs who have no nutritional science background. The aim of this study was to evaluate the accuracy and ease-of-use of MenuCal.

The accuracy of MenuCal was tested using recipes ( $n$ 750) reflective of those sold in FBs in Ireland. The recipes were analysed by trained nutritionists using the MenuCal system to provide the 'benchmarked recipes' (ingredient weights and calories). The testing was conducted in two phases by catering students and FBs, with refinements made to the system following phase 1 testing. For both phases, accuracy was determined by the $\%$ difference for recipe ingredient weights and calorie content. An evaluation questionnaire was completed to assess ease-of-use.

During phase 1, student participants ( $n$ 75) entered a total of 525 recipes. When compared with the benchmarked recipes, the overall median difference for both calories and weight was $0 \cdot 5 \%$ and $0 \cdot 13 \%$ respectively. Eighty-six percent and $81 \%$ of recipes differed from the corresponding benchmarked recipes, with the error for half of the recipes ranging from- $12 \%$ to $+12 \%$ for calories and $-5 \%$ to $+13 \%$ for weight. The differences were mainly due to portion size, selection of different ingredients (e.g. raw beef instead of cooked beef) and typing errors (e.g. 5,000 g instead of $500 \mathrm{~g}$ ). In phase 2, FBs $(n 54)$ entereda further 159 recipes and the median $\%$ calorie and weight difference was zero and 1\% respectively. Similar to phase $1,89 \%$ and $87 \%$ of recipes varied from the corresponding benchmarked recipes, with the error for half of the recipes in the order of $-14 \%$ to $+12 \%$ for calories and $-6 \%$ to $+15 \%$ for weight. The main reason for the discrepancies in \% calorie and weight was due to difference in portion size due to limited information being provided in the recipe (e.g. 2 potatoes in the recipe rather than $150 \mathrm{~g}$ potatoes, i.e. portion size estimation variations).

MenuCal was rated by $84 \%$ of students and $86 \%$ of FBs as an 'easy' to 'very easy' system to use. Furthermore, $88 \%$ of students and $84 \%$ of FBs indicated they were 'likely' or 'very likely' to use MenuCal when launched. Participants requested more information on calculating weight loss/gain on cooking, simplification of food descriptions and incorporation of branded products used in the catering industry to make MenuCal more user-friendly.

In conclusion, MenuCal was found by FBs and catering students to be a user-friendly system for estimating the calorie content of menu items. The benchmarking procedure demonstrated good agreement between the trained nutritionists and participants for the calorie content of the recipes provided. This study identified the areas where users require additional support, portion size guidance and simpler food descriptions to increase the accuracy of MenuCal.

1. Block JP, Condon SK, Kleinman K et al. (2013) BMJ, [Epub ahead of print].

2. Food Safety Authority of Ireland (2012). Calories on Menus in Ireland - Report on a national consultation at: http://www.fsai.ie/WorkArea/ DownloadAsset.aspx?id=11419 